\title{
Brachial-ankle Pulse Wave Velocity and Renal Function can Predict Coronary Artery Disease in Asymptomatic Japanese Patients with Type 2 Diabetes
}

\author{
Yoko Matsuzawa ${ }^{1}$, Kazuhiko Yumoto ${ }^{2}$, Kenichi Kato ${ }^{2}$, Jun Saito ${ }^{1}$, Masao Omura ${ }^{1}$ and Tetsuo Nishikawa ${ }^{1 *}$ \\ ${ }^{1}$ Endocrinology \& Diabetes Center, Yokohama Rosai Hospital, Yokohama City, Japan \\ ${ }^{2}$ Division of Cardiology, Department of Medicine, Yokohama Rosai Hospital, Yokohama City, Japan
}

\begin{abstract}
Received: April 07, 2015; Accepted: June 11, 2015; Published: June 25, 2015
*Corresponding author: Tetsuo Nishikawa, Endocrinology and Diabetes Center, Yokohama Rosai Hospital 3211 Kozukue-cho, Kohoku-ku, Yokohama City, Kanagawa 222-0036, Japan, Tel: 81-454748111; Fax: 81-454748323; E-mail: tetsuon@yokohamah.rofuku.go.jp
\end{abstract}

\begin{abstract}
In patients with diabetes mellitus, myocardial perfusion defects are often observed in the absence of obstructive Coronary Artery Disease (CAD), thereby presenting a diagnostic problem. Meanwhile, the indications for Multidetector Computed Tomography (MDCT) in the early diagnosis of CAD in diabetic patients has not yet been clarified. We performed MDCT with written informed consent on 74 type 2 diabetic patients ( 57 men and 17 women, mean age $57.6 \pm 10.4$ years, mean body mass index $25.8 \pm 4.9 \mathrm{Kg} / \mathrm{m} 2$, mean glycosylated hemoglobin [HbA1c] $7.2 \pm 1.4 \%$, smokers $39.2 \%$ ) with no symptoms or history of CAD. Blood pressure and low-density lipoprotein cholesterol were well controlled. Patients treated with insulin or those who had serum creatinine levels $>1.0 \mathrm{mg} / \mathrm{dl}$ were excluded, and all patients had a negative exercise tolerance stress test. Only 17 patients $(23.0 \%)$ were found to have normal coronary arteries, with neither plaque nor calcification. Coronary plaques were found in 26 patients (35.1\%), and calcification was found in 25 (33.8\%). MDCT angiography also demonstrated significant ( $>50 \%$ ) stenosis in 21 of 74 cases $(28.4 \%)$. Various clinical parameters were compared between patients with obstructive CAD who showed significant (> $50 \%$ ) stenosis and non-obstructive CAD patients without significant stenosis. The obstructive CAD group had significantly greater age, and higher serum creatinine levels, brachial Pulse Wave Velocity (PWV), and carotid artery intima-media thickness evaluated by ultrasonography. HbA1c, waist circumference, visceral fat area, cholesterol, and blood pressure were not significantly different between the groups. In the multivariate analysis, PWV, plaque score, and serum creatinine were independent factors for estimating the presence of advanced coronary lesions.
\end{abstract}

\section{Introduction}

The impact of diabetes on cardiovascular disease is further illustrated by the fact that the risk of developing a myocardial infarction in diabetic patients without known heart disease is equivalent to the risk observed in non-diabetic survivors of a prior infarction [1]. Because of the high incidence of coronary artery disease (CAD) among diabetic patients, the need for accurate and early identification is paramount. Computed tomography (CT) angiography received a boost recently with the availability of the 64-slice CT scanner. The latest generation of CT scanners can provide exquisite imaging of cardiac structures, coronary plaque morphology data, and details of atherosclerotic lesions, quickly and with minimal patient discomfort or risk. A recent Japanese observational study demonstrated that serum triglyceride level was a leading predictor of Coronary Heart Disease (CHD), comparable to low-density lipoprotein cholesterol in Japanese patients with type 2 diabetes [2]. The authors suggested that ethnic group-specific strategies for prevention of diabetic macroangiopathy may be indicated, because the serum triglyceride level is not a leading predictor of CHD in diabetic subjects in Western countries [2]. Moreover, the most common reported comorbidities associated with chronic kidney disease are hypertension, retinopathy, CHD, myocardial infarction, and congestive heart failure, according to a study from Kidney Disease Improving Global Outcomes (KDIGO) 2013 [3]. Even mildly reduced estimated glomerular filtration rate $\left(<75 \mathrm{ml} / \mathrm{min} / 1.73 \mathrm{~m}^{2}\right)$ increased the risk of CHD incidence among type 2 diabetes patients [4]. The objectives of this study were to determine the prevalence and severity of coronary atherosclerosis in asymptomatic subjects with type 2 diabetes, since asymptomatic CHD, with $\geq 50 \%$ diameter stenosis and myocardial perfusion abnormalities, was reportedly detected in more than half of Japanese patients with type 2 diabetes with vascular complications [5]. Moreover, it was reported that plasma adiponectin levels are an independent predictor of CHD in Caucasian men initially free of CHD [6], and a risk calculator including albuminuria enhances CHD prediction, compared to a calculator using only standard risk factors, in men and women [7]. We also sought to determine which clinical parameter(s), such as pulse wave velocity (PWV), plaque score, and renal function, which are related to diabetic complications, account for the findings on cardiovascular CT angiography.

\section{Methods}

We undertook a retrospective review of 74 well-controlled, adult type 2 diabetes patients who underwent cardiovascular 
CT angiography at Yokohama Rosai Hospital between December 2009 and June 2011. All subjects met the following criteria: 1) no symptoms or past history of CAD, 2) not taking insulin, and 3) serum creatinine $\leq 1.0 \mathrm{mg} / \mathrm{dl}$. Thus, type 1 diabetic patients were not included in the study. Patients included in this study were referred to the Heart Center of Yokohama Rosai Hospital by specialists of the Endocrinology \& Diabetes Center in our hospital, for diagnostic angiography for unsuspected CAD.

All subjects underwent coronary CT angiography using the Siemens Sensation (Siemens Healthcare, Tokyo) Cardiac 64-Slice CT. Cross-sectional images were reconstructed with a slice thickness of $0.75 \mathrm{~mm}$ at $0.4 \mathrm{~mm}$ intervals, with the use of an electrocardiogram gated half-scan reconstruction algorithm [8], to obtain an image acquisition window of $164 \mathrm{~ms}$. All vessels $>1.5$ $\mathrm{mm}$ were evaluated. Patients with a resting heart rate $>60$ beats per minute were intravenously medicated with $5 \mathrm{mg}$ metoprolol to enhance image quality and reduce distortion. A heart rate of 60 beats per minute or less critically influences image quality and is considered a desirable threshold to minimize motion artifacts [9]. Disease status was categorized as follows: no disease, nonobstructive CAD (one or more coronary lesions with $<50 \%$ stenosis), and obstructive CAD (one or more lesions > 50\%) Brachial-ankle pulse wave velocity (baPWV) was measured by its apparatus (Kohrin Co, Tokyo) [10]. Adiponectin was measured by enzyme-linked immunosorbent assay (Otsuka Pharmaceutical Co, Tokyo, Japan). Levels of HbA1c were determined by using the HA-8170 apparatus (Arkray, Kyoto, Japan), which employs a regular ion exchange HPLC method for quantification of this glycohemoglobin, and laboratory parameters of serum creatinine, cholesterol, triglycerides and other variables were analyzed in a Nippon Denshi Autoanalyzer (JCA-RX20, Tokyo, Japan) as previously reported [11]. Each parameter, including visceral and subcutaneous fat area determined by abdominal CT scan, and homeostasis model assessment of insulin resistance (HOMA-IR), was determined, as previously reported elsewhere [11,12].

Descriptive statistics (frequency distributions and mean values) were completed for each patient cohort. All values are presented as the mean \pm standard deviation. Statistical significance was evaluated among the three cohorts using oneway analysis of variance. Differences were considered to be statistically significant at a $P$ value of $<0.05$.

\section{Results}

\section{Patient profiles}

The clinical characteristics of the subjects, including body weight, waist circumference, visceral and subcutaneous fat area determined by abdominal CT scan, blood pressure, baPWV, serum cholesterol, serum creatinine, fasting glucose, HOMA-IR, and glycosylated hemoglobin (HbA1c), are shown in Table 1a and b.

\section{MDCT angiography findings}

In this study, MDCT angiography showed a high prevalence (77.0\%) of CAD among asymptomatic type 2 diabetic patients (Table 2). Only 17 patients (23\%) had normal coronary arteries, with neither plaque nor calcification. Coronary plaques were found in 26 patients, and calcification was found in 25. MDCT angiography also demonstrated significant (> 50\%) stenosis in 21 of 74 cases $(28.4 \%)$.

Patients with significant stenosis were classified as the obstructive CAD group ( $\mathrm{n}=21)$, and the others were included in the non-obstructive group ( $\mathrm{n}=53)$, including 17 cases with normal coronary arteries.

Obstructive CAD was found in $28.4 \%$ of patients, and 3 cases (4.1\%) required percutaneous coronary intervention for left anterior descending disease.

\section{Comparison of each parameter between patients with non-obstructive CAD and those with obstructive CAD}

The obstructive CAD group had significantly greater age, and higher serum creatinine, cystatin $\mathrm{C}$, and maximum Intima-Media Thickness (IMT) by ultrasonography of the carotid artery, while estimated glomerular filtration rate was significantly lower in the patients with obstructive CAD (Table 3).

Table 1a: Background of the subjects.

\begin{tabular}{|c|c|c|c|}
\hline Variatbles & $\mathbf{N}(\mathbf{\%})$ & Variables & Mean \pm SD \\
\hline Smoking & & Age(years) & $57.6 \pm 10.4$ \\
\hline non-smoker & $25(33.8 \%)$ & $\begin{array}{c}\text { Duration of diabetes } \\
\text { (years) }\end{array}$ & $7.68 \pm 6.38$ \\
\hline smoker & $29(39.2 \%)$ & BMI $\left(\mathrm{kg} / \mathrm{m}^{2}\right)$ & $26.3 \pm 4.1$ \\
\hline ex-smoker & $18(24.3 \%)$ & Waist circumferrence $(\mathrm{cm})$ & $92.0 \pm 11.8$ \\
\hline unknown & $2(2.7 \%)$ & Visceral fat area $\left(\mathrm{cm}^{2}\right)$ & $116.6 \pm 45.8$ \\
\hline & & Subctaneous fat area $\left(\mathrm{cm}^{2}\right)$ & $200.7 \pm 106.8$ \\
\hline
\end{tabular}

Seventy-four type 2 diabetic patients (57 men and 17 women) were recruited to the study with written informed consent. All subjects met the following criteria: 1) no symptoms or past history of CAD, 2) not taking insulin, and 3) serum creatinine $\leq 1.0 \mathrm{mg} / \mathrm{dl}$. Medications: 59 (79.7\%) patients were on oral antihyperglycemic drugs, 38 (51.4\%) were on an Angiotensin-Converting Enzyme Inhibitor (ACEI) or AngiotensinReceptor Blocker (ARB), and 50 (67.6\%) were on statins. Volume of visceral and subcutaneous fat area was estimated as described in detail in the Text.

Table 1b: Laboratory Findings.

\begin{tabular}{|c|c|c|c|}
\hline Variables & Mean \pm SD & Variables & Mean \pm SD \\
\hline FPG (mg/dl) & $136.5 \pm 47.9$ & $\begin{array}{c}\text { Diastolic BP } \\
\text { (mmHg) }\end{array}$ & $72.5 \pm 9.5$ \\
\hline HbA1c (\%) & $7.24 \pm 1.45$ & TG (mg/dl) & $126.1 \pm 72.3$ \\
\hline $\begin{array}{c}\text { Glycoalbumin } \\
(\%)\end{array}$ & $19.3 \pm 4.3$ & LDL-Chol (mg/dl) & $103.0 \pm 23.2$ \\
\hline $\begin{array}{c}\text { Fasting IRI ( } \mu \mathrm{U} / \\
\text { ml) }\end{array}$ & $7.58 \pm 4.51$ & HDL-Chol $(\mathrm{mg} / \mathrm{dl})$ & $48.7 \pm 13.2$ \\
\hline HOMA-IR & $2.66 \pm 1.99$ & LDL/HDL ratio & $2.29 \pm 0.85$ \\
\hline $\begin{array}{c}\text { Systolic BP } \\
\text { (mmHg) }\end{array}$ & $124.1 \pm 12.3$ & $\begin{array}{r}\text { Adiponectin }(\mu \mathrm{\mu g} / \\
\text { dl) }\end{array}$ & $9.69 \pm 6.07$ \\
\hline
\end{tabular}

FPG = Fasting Plasma Glucose, IRI = Immunoreactive Insulin, BP = Blood Pressure, $\mathrm{TG}=$ Triglycerides, LDL-Chol = Low-Density Lipoprotein Cholesterol, HDL-Chol = High-Density Lipoprotein Cholesterol, BMI = Body Mass Index. Each parameter was determined as described in detail in the Text. 
HbA1c, waist circumference, visceral fat area, cholesterol, and blood pressure were not significantly different between the groups. There were no significant differences in BMI, urinary albumin excretion, and plasma levels of glycoalbumin, fasting insulin, creatinine, and adiponectin between the two groups. In the multivariate analysis, baPWV, max IMT and serum creatinine proved to be independent factors for estimating the existence of advanced coronary lesions (Table 4).

We also analyzed a Receiver Operating Characteristic (ROC) curve for obstructive CAD, using parameters such as age, maximum IMT, baPWV, and serum Cre level (Figure 1). The cutoff point for predicting obstructive CAD was more than 1550

Table 2: Prevalence of coronary artery disease in asymptomatic type 2 diabetic patients.

\begin{tabular}{|c|c|}
\hline Variables & N (\%) \\
\hline Obstructive CAD & $21(28.4 \%)$ \\
\hline Non-obstructive CAD & $36(48.6 \%)$ \\
\hline Normal MDCT & $17(23.0 \%)$ \\
\hline
\end{tabular}

$\mathrm{CAD}=$ Coronary Artery Disease, MDCT $=$ Multidetector Computed Tomography

MDCT was performed as described in detail in the Text.

Table 3: Comparison of each Parameter between Patients with NonObstructive CAD and those with Obstructive CAD.

\begin{tabular}{|c|c|c|c|}
\hline & $\begin{array}{l}\text { Obstructive CAD } \\
(-) n=53\end{array}$ & $\begin{array}{c}\text { Obstructive } \\
\text { CAD(+) n=21 }\end{array}$ & P value \\
\hline & Mean \pm SD & Mean \pm SD & \\
\hline Age (years) & $56.0 \pm 10.7$ & $61.9 \pm 8.5$ & $0.026^{*}$ \\
\hline $\begin{array}{l}\text { Duration of diabetes } \\
\text { (years) }\end{array}$ & $6.9 \pm 6.8$ & $9.8 \pm 4.5$ & 0.083 \\
\hline BMI $\left(\mathrm{kg} / \mathrm{m}^{2}\right)$ & $26.6 \pm 4.0$ & $25.6 \pm 4.3$ & 0.349 \\
\hline $\begin{array}{l}\text { Visceral Fat area } \\
\left(\mathrm{cm}^{2}\right)\end{array}$ & $115.4 \pm 43.7$ & $120.3 \pm 53.1$ & 0.716 \\
\hline $\begin{array}{l}\text { Subcutaneous fat } \\
\text { area }\left(\mathrm{cm}^{2}\right)\end{array}$ & $197.9 \pm 88.6$ & $209.6 \pm 154.6$ & 0.705 \\
\hline FPG (mg/dl) & $132.1 \pm 48.9$ & $147.5 \pm 44.4$ & 0.216 \\
\hline HbA1c (\%) & $7.13 \pm 1.09$ & $7.52 \pm 0.88$ & 0.146 \\
\hline Glycoalbumin (\%) & $18.7 \pm 4.6$ & $20.8 \pm 3.0$ & 0.053 \\
\hline Fasting IRI $(\mu \mathrm{U} / \mathrm{ml})$ & $7.34 \pm 4.14$ & $8.19 \pm 5.40$ & 0.478 \\
\hline HOMA-IR & $2.43 \pm 1.68$ & $3.27 \pm 2.59$ & 0.111 \\
\hline Systolic BP (mmHg) & $122.3 \pm 12.3$ & $128.5 \pm 11.7$ & 0.053 \\
\hline Diastolic BP (mmHg) & $71.4 \pm 9.2$ & $75.4 \pm 9.8$ & 0.101 \\
\hline TG (mg/dl) & $130.0 \pm 74.5$ & $116.3 \pm 67.1$ & 0.464 \\
\hline LDL-Cho (mg/dl) & $103.2 \pm 22.9$ & $102.6 \pm 24.7$ & 0.918 \\
\hline HDL-Cho (mg/dl) & $48.6 \pm 13.1$ & $48.9 \pm 13.8$ & 0.926 \\
\hline Adiponectin (mg/dl) & $8.40 \pm 5.43$ & $10.80 \pm 7.28$ & 0.127 \\
\hline Cre (mg/dl) & $0.73 \pm 0.16$ & $0.80 \pm 0.12$ & 0.074 \\
\hline eGFR & $83.6 \pm 16.6$ & $73.5 \pm 11.1$ & $0.013^{*}$ \\
\hline U-Alb (mg/gCre) & $38.5 \pm 91.2$ & $50.3 \pm 67.7$ & 0.593 \\
\hline Cystatin C (mg/L) & $0.91 \pm 0.15$ & $1.00 \pm 0.16$ & 0.019 \\
\hline Max IMT (mm) & $1.39 \pm 0.64$ & $2.11 \pm 1.25$ & $0.002^{* *}$ \\
\hline baPWV (cm/s) & $1500.0 \pm 283.4$ & $1825.6 \pm 326.3$ & $0.000^{* *}$ \\
\hline
\end{tabular}

Results were expressed as mean \pm SD. maxIMT; maximum intimamedia thickness, baPWV; brachial pulse wave velocity, eGFR; estimated glomerular filtration rate, U-Alb; urinary albumin excretion rate. Each parameter was determined as described in detail in the Text.
Table 4: Multivariate Logistic Analysis of Clinical Characteristics.

\begin{tabular}{|l|c|c|c|}
\hline & Odds ratio & $\mathbf{9 5 \%}$ CI & $\boldsymbol{P}$ Value \\
\hline baPWV & 3.985 & $1.795-8.850$ & 0.001 \\
\hline Cre & 2.106 & $1.052-4.215$ & 0.036 \\
\hline Max IMT & 1.152 & $1.152-4.930$ & 0.019 \\
\hline
\end{tabular}

baPWV, max IMT, and serum creatinine (Cre) were significantly related to the presence of obstructive CAD. Age and systolic blood pressure were not significantly related to obstructive CAD. baPWV = brachial-ankle pulse wave velocity, IMT = Intima-Media Thickness

$\mathrm{cm} / \mathrm{s}$ of baPWV (sensitivity: 0.90; specificity: 0.68 ). In patients with more than $1550 \mathrm{~cm} / \mathrm{s}$ of baPWV, serum Cre level was then the best predictor among the parameters (Cre $\pm 0.6 \mathrm{mg}$ / dl, sensitivity: 0.84; specificity: 0.82) (Figure 2). Thus the most useful clinical parameters for predicting obstructive CAD, as determined from the ROC analysis, were as follows: baPWV > $1550 \mathrm{~cm} / \mathrm{s}$ and serum creatinine $>0.60 \mathrm{mg} / \mathrm{dl}$.

\section{Discussion}

Our review is the first to evaluate the increased coronary atheromatous burden in patients with type 2 diabetes by MDCT, and provides interesting insights into the characteristics of the disease in these high-risk populations. Our data clearly demonstrated that there is a high prevalence of CAD in type 2 diabetic patients who are asymptomatic and have a negative exercise electrocardiogram. CAD events can be prevented by comprehensive treatment of various risk factors, such as hypertension and dyslipidemia [13]. However, the study patients were already well-controlled for hypertension, dyslipidemia, and hyperglycemia by treatment with angiotensin receptor blockers, statins, and oral hypoglycemic agents.

Our data demonstrated that there was no significant difference in BMI and visceral fat area between the obstructive and non-obstructive CAD groups. On the other hand, a study consisting of 2842 men and 3196 women, the FINRISK '92 survey [14], reported that body fat percentage measured by a near-infrared interactance device was a significant predictor of cardiovascular disease (CVD) and CHD events among men and women, but did not provide any additional predictive power over and above simpler measures, such as BMI or waist-to-hip ratio. It was also reported that basal C-peptide is related to cardiovascular predictors (IMT) of type 2 diabetes in Korean patients, suggesting that basal C-peptide does provide a further indication of CVD [15]; however, our data demonstrated that insulin resistance, estimated by HOMA-IR and fasting levels of serum insulin, did not differ between the obstructive and non-obstructive CAD groups. A prospective cohort study of 7067 Chinese type 2 diabetic patients without a history of CHD, enrolled from 1995 to 2005, demonstrated that in type 2 diabetes, albuminuria links conventional risk factors and CHD [16]. The onset of chronic kidney disease changes risk associations between lipids and CHD [16], while there was no significant difference in albuminuria between the obstructive and non-obstructive CAD groups in our study. It was previously reported that higher baPWV could be an independent risk factor for future microalbuminuria in patients 


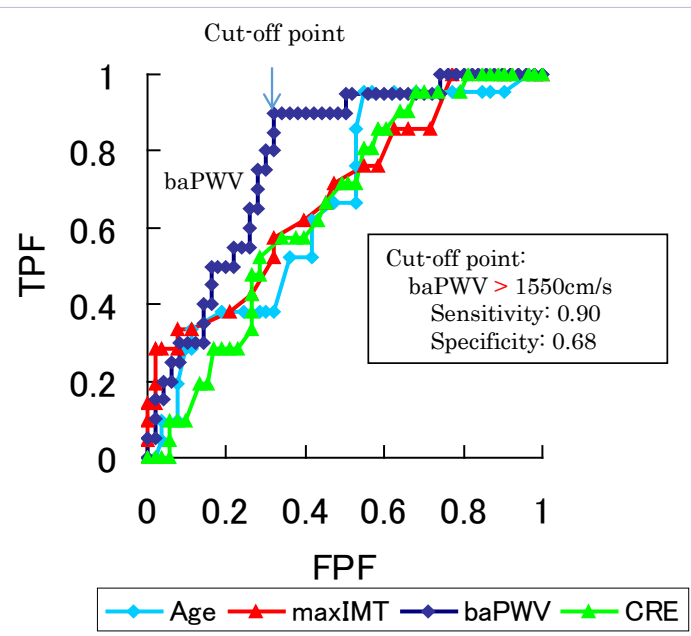

Figure 1: ROC curve for obstructive CAD.

Receiver operating characteristic (ROC) curve analysis for obstructive coronary artery disease (CAD) ( $n=70$ cases) was performed using parameters such as age, maximum intima-media thickness (IMT), brachial-ankle pulse wave velocity (baPWV), and serum creatinine (Cre) level.

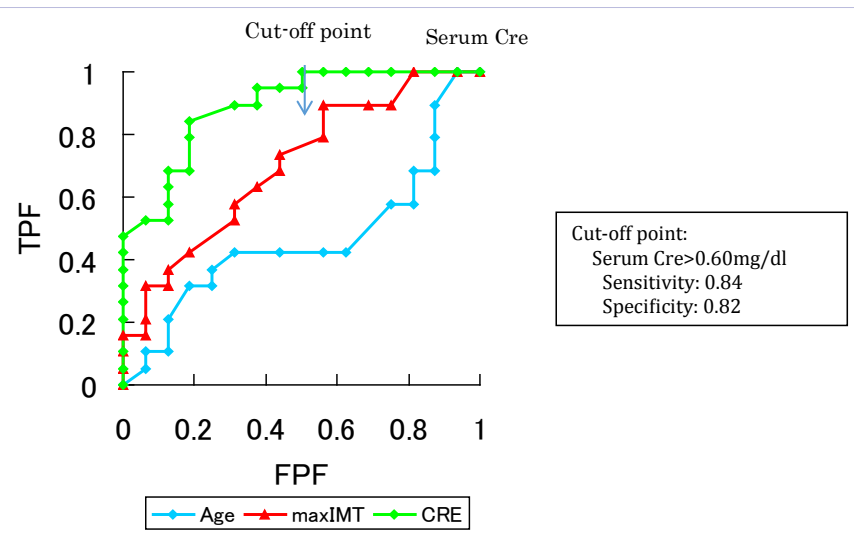

Figure 2: ROC curve for obstructive CAD in patients with baPWV > 1550 $\mathrm{cm} / \mathrm{s}$.

Receiver operating characteristic (ROC) curve analysis for obstructive coronary artery disease (CAD) in patients with brachial-ankle pulse wave velocity (baPWV) $>1550 \mathrm{~cm} / \mathrm{s}(\mathrm{n}=25$ cases) was performed using parameters such as age, maximum intima-media thickness (IMT), and serum creatinine (Cre) levels.

with essential hypertension [17], suggesting that baPWV may reflect future microalbuminuria and CHD.

Cases with asymptomatic type 2 diabetes were subjected to the Diabetes Audit and Research in Tayside, Scotland, suggesting that age at diagnosis, duration of diabetes, HbA1c, smoking (current, past, never), sex, systolic blood pressure, treated hypertension, total cholesterol, and height seem to be the significant predictors for future cardiovascular events [18]. Thus, our data are consistent with the previous findings of the effect of age [18] on obstructive CAD.

It was also reported that baPWV is an independent risk factor for future cardiovascular events in patients with essential hypertension [19]. In the present study, baPWV and serum creatinine proved to be the most useful clinical markers for predicting obstructive CAD. MDCT and/or coronary angiography are highly recommended when patients meet the criteria of baPWV $>1550 \mathrm{~cm} / \mathrm{s}$ and Cre $>0.60 \mathrm{mg} / \mathrm{dl}$.

It was reported that baPWV was significantly and positively associated with age, systolic blood pressure, and the Framingham risk score in hypertensive patients, and also that the areas under the ROCs of PWV can predict the presence of both stroke and CAD [10], while multivariate analysis with the Cox proportional hazards model identified IMT, but not baPWV, as a significant determinant of CVD [10]. Another report demonstrated that the combination of the Framingham Risk Score with IMT rather than with baPWV improved the prediction of CVD [20]. Moreover, our data clearly suggest that we can predict the risk and possibility of obstructive CAD by examining baPWV and serum creatinine levels in asymptomatic patients with type 2 diabetes.

In conclusion, HbA1c and Glycoalbumin (GA) levels with fasting insulin levels do not always reflect the risk of obstructive CAD. The present data also demonstrated that adiponectin concentration and visceral fat area were not useful markers for predicting obstructive CAD. Finally, the present data clearly demonstrated that we can predict the risk and possibility of obstructive CAD by examining baPWV and serum creatinine levels in asymptomatic patients with type 2 diabetes. On the other hand, the present study was performed in a limited number of asymptomatic type 2 diabetic patients. Moreover, we did not follow up these patients for future cardiovascular events. Thus, further studies are needed on two separate groups, derived from the asymptomatic type 2 diabetic patients according to the values for baPWV and serum creatinine as described above, with prospective monitoring for the presence of obstructive CAD.

\section{References}

1. Haffner SM, Lehto S, Ronnemaa T, Pyroala K, Laakso M. Mortality from coronary heart disease in subjects with type 2 diabetes and in nondiabetic subjects with and without prior myocardial infarction. N Engl J Med. 1998; 339(4):229-334.

2. Sone H, Tanaka S, Tanaka S, Iimuro S, Oida K, Yamasaki Y, et al. Serum level of triglycerides is a potent risk factor comparable to LDL cholesterol for coronary heart disease in Japanese patients with type 2 diabetes: subanalysis of the Japan Diabetes Complications Study Group (JDCS). J Clin Endocrinol Metab. 2011; 96(11):3448-3456. doi: 10.1210/jc.2011-0622.

3. Bailey RA, Wang Y, Zhu V, Rupnow MF. Chronic kidney disease in US adults with type 2 diabetes: an updated national estimate of prevalence based on Kidney Disease: Improving Global Outcomes (KDIGO) staging. BMC Res Notes. 2014 2; 7:415. doi: 10.1186/17560500-7-415.

4. Wang Y, Katzmarzyk PT, Horswell R, Zhao W, Johnson J, Hu G. Kidney function and the risk of cardiovascular disease in patients with type 2 diabetes. Kidney Int. 2014; 85(5):1192-1199. doi: 10.1038/ ki.2013.396.

5. Tsujimoto T, Kajio H, Takahashi Y, Kishimoto M, Noto H, YamamotoHonda R, et al. Asymptomatic coronary heart disease in patients with type 2 diabetes with vascular complications: a cross-sectional study. 
BMJ Open 2011; E000139. Doi 10.1136/bmjopen-2011-000139.

6. Ai M, Otokozawa S, Asztalos BF, White CC, Cupples LA, Nakajima K, et al. Adiponectin: an independent risk factor for coronary heart disease in men in the Framingham offspring Study. Atherosclerosis. 2011; 217(2):543-8. doi: 10.1016/j.atherosclerosis.2011.05.035.

7. Shara NM, Wang H, Valaitis E, Pehlivanova M, Carter EA, Resnick $\mathrm{HE}$, et al. Comparison of estimated glomerular filtration rates and albuminuria in predicting risk of coronary heart disease in a population with high prevalence of diabetes mellitus and renal disease. Am J Cardiol. 2011; 107(3):399-405. doi: 10.1016/j.amjcard.2010.09.036.

8. Flohr T, Bruder H, Stierstorfer K, Simon J, Schaller S, Ohnesorge B, et al New technical developments in multislice CT, part 2; sub-millimeter 16-slice scanning and increased rotational speed for cardiac imaging. Rofo. 2002; 174(8):1022-1027.

9. Schroeder S, Kopp AF, Kuettner A, Burgstahler C, Herdeg C, Heuschmid $\mathrm{M}$, et al. Influence of heart rate on vessel visibility in noninvasive coronary angiography using new multislice computed tomography: experience in 94 patients. Clin Imaging. 2002; 26(2):106-111.

10. Tanaka H, Munakata M, Kawano Y, Ohishi M, Shoji T, Sugawara J, et al. Comparison between carotid-femoral and brachial-ankle pulse wave velocity as measures of arterial stiffness. J Hypertens. 2009; 27(10):2022-2027. doi: 10.1097/HJH.0b013e32832e94e7.

11. Soyama A, Nishikawa T, Ishizuka T, Ito H, Saito J, Yagi K, et al. Clinical usefulness of the thickness of preperitoneal and subcutaneous fat Layer in the abdomen estimated by ultrasonography for diagnosing abdominal obesity in each type of impaired glucose tolerance in man. Endocr J. 2005; 52(2):229-236.

12. Matsuzawa Y, Sakura K, Saito J, Omura M, Nishikawa T. Salivary Cortisol Can Reflect Adiposity and Insulin Sensitivity in Type 2 Diabetes Steroids - Clinical Aspect, Edited by Prof. Hassan Abduljabbar, 142 150. DOI: $10.5772 / 27172$. InTech, Croatia

13. Wong ND, Patao C, Malik S, Iloeje U. Preventable coronary heart disease events from control of cardiovascular risk factors in US adults with diabetes (projections from utilizing the UKPDS risk engine). Am J Cardiol. 2014; 113(8):1356-1361. doi: 10.1016/j. amjcard.2013.12.042.

14. Pajunen P, Jousilahti P, Borodulin K, Harald K, Tuomilehto J, Salomaa V. Body fat measured by a near-infrared interactance device as a predictor of cardiovascular events: the FINRISK'92 cohort. Obesity (Silver Spring). 2011; 19(4):848-852. doi: 10.1038/oby.2010.236.

15. Kim ST, Kim BJ, Lim DM, Song IG, Jung JH, Lee KW, et al. Basal C-peptide Level as a Surrogate Marker of Subclinical Atherosclerosis in Type 2 Diabetic Patients. Diabetes Metab J. 2011; 35(1): 41-49. doi: 10.4093/ dmj.2011.35.1.41.

16. Yang X, Ma RC, So WY, Ko GT, Kong AP, Lam CW, et al. Impacts of chronic kidney disease and albuminuria on associations between coronary heart disease and its traditional risk factors in type 2 diabetic patients - the Hong Kong diabetes registry. Cardiovasc Diabetol. 2007; 6:37.

17. Munakata M, Miura Y, Yoshinaga K; J-TOPP study group. Higher brachial-ankle pulse wave velocity as an independent risk factor for future microalbuminuria in patients with essential hypertension: the J-TOPP study. J Hypertens. 2009; 27(7):1466-1471. doi: 10.1097/ HJH.0b013e32832b4740.

18. Donnan PT, Donnelly L, New JP, Morris AD. Derivation and validation of a prediction score for major coronary heart disease events in a U.K. type 2 diabetic population. Diabetes Care. 2006; 29(6):1231-1236.

19. Munakata M, Konno S, Miura Y, Yoshinaga K; J-TOPP study group. (2012) Prognostic significance of the brachial-ankle pulse wave velocity in patients with essential hypertension: final results of the J-TOPP study. Hypertens Res. 2012; 35(8):839-842. doi: 10.1038/ hr.2012.53.

20. Yoshida M, Mita T, Yamamoto R, Shimizu T, Ikeda F, Ohmura C, et al. Combination of the Framingham risk score and carotid intimamedia thickness improves the prediction of cardiovascular events in patients with type 2 diabetes. Diabetes Care. 2012; 35(1):178-180. doi: $10.2337 /$ dc11-1333. 